\title{
TWENTY-NINTH ANNUAL SASKATCHEWAN CHRISTMAS BIRD COUNT, 1970
}

\author{
Compiled by Mary Houston, 863 University Drive, Saskatoon
}

Observers in 35 areas (33 in 1969), plus one in the Northwest Territories, participated in the 1970 Saskatchewan Christmas bird count. Sixty species of birds were observed on count days, with three additionals seen, not on a count day, but during the period between December 22 and January 3. A Red-shafted Flicker reported from Regina, added one new species to the all-time list, bringing the total for the 29 years of Saskatchewan counts to 114 species (with six additionals).

An unusual number of Robins was recorded throughout the province. Although they have been reported in 21 of the 29 years, they have never been so widespread or so numerous. Nine of the 34 localities reported Robins during count period. In Saskatoon four of the 10 parties reported Robins-one group sighting a flock of 30. There is certainly no shortage of berries for them.

The noticeably low species were Pine Grosbeak, reported in only six areas and the usually ubiquitous Redpolls, reported from only four. Whitewinged Crossbills were seen only at Cumberland House, and Red Crossbills were not reported at all.

ABERDEEN-SMUTS, Jan. 2; 56 miles by car in $3 \frac{1}{2}$ party hours; 3 species, 359 individuals. Black-billed Magpie, 16; House Sparrow, 102; Snow Bunting, 241.-Dr. and Mrs. J. B. Gollop.

BORDEN BRIDGE, Dec. 27; 30 miles by car and 1 mile on foot in 3 hours; temp. $2^{\circ}$, clear; 7 species, 443 individuals. Ruffed Grouse, 3; Sharp-tailed Grouse, 8; Blue Jay, 1; Black-billed Magpie, 10; Black-capped Chickadee, 4; House Sparrow, 16; Snow Bunting, L.01.-John and Stanley Shadick.

BROADVIEW, Dec. 30 ; 36 miles by car and 3 miles on foot in 4 hours; temp. $20^{\circ}$; wind S.W. $15 \mathrm{mph} ; 9$ species, 243 individuals. Sharp-tailed
Grouse, 11; Gray Partridge, 16; Great Horned Owl, 1; Hairy Woodpecker, 2; Downy Woodpecker 3; Black-billed Magpie, 4; Black-capped Chickadee, 11; House Sparrow, 66; Snow Bunting, 129. (Add: Northern Shrike, 1, Jan. 1.) David Chaskavich and Donald Weidl.

BUFFALO LAKE, Eastern end, Dec. 29 ; 12 miles on foot and 4 miles by car; temp. $25^{\circ}$; wind $15 \mathrm{mph}$; 3 species, 15 individuals. Black-billed Magpie, 3; Black-capped Chickadee, 10; Goldencrowned Kinglet, 2.-John Horton, Pat Kern, David Robinson, (Leith Knight, compiler).

CRESTWYND, Dec. $27 ; \frac{1}{2}$ mile on foot and 25 miles by car; temp. $-8^{\circ}$; wind ESE $18 \mathrm{mph}$; cloudy; 7 species, 264 individuals. Sharp-tailed Grouse, 1; Rock Dove, 20; Snowy Owl, 1; Shorteared Owl, 1; Black-billed Magpie, 1; House Sparrow, 15; Snow Bunting, 225. John Horton, David Robinson, (Leith Knight, compiler).

CUMBERLAND HOUSE, Jan. $2 ; 6$ miles on foot in $5 \frac{1}{2}$ party hours and 27 miles by car in 12 party hours; temp. $6^{\circ}$ to $10^{\circ}$; wind NW $10-15 \mathrm{mph}$; overcast, 13 inches of snow; 21 species, 272 individuals. Goshawk, 3; Ruffed Grouse, 1; Willow Ptarmigan, 3; Sharp-tailed Grouse, 19; Great Horned Owl, 1; Hairy Woodpecker, 1; Downy Woodpecker, 1; Gray Jay, 1; Blue Jay, 5; Black-billed Magpie, 13; Common Raven, 21; Black-capped Chickadee, 18; Red-breasted Nuthatch, 2; Bohemian Waxwing, 11; House Sparrow, 50; Brewer's Blackbird, 1; Evening Grosbeak, 4; Pine Grosbeak, 25; Whitewinged Crossbill, 6; Snow Bunting, 65. - Vern Gunnlaugson, Dr. and Mrs. Stuart Houston (compilers), David and Donald Houston, Stanley D. Riome, Stanley J. Shadick.

DILKE, Dec. 28; 82 miles by car in 4 hours; temp. $10^{\circ}$; wind SSE 5-10 mph; sunny; 18" snow heavily drifted; 9 
species, 211 individuals. Sharp-tailed Grouse, 6; Gray Partridge, 37; Rock Dove, 16; Great Horned Owl, 1; Snowy Owl, 1; Black-billed Magpie, 30; Northern Shrike, 1; House Sparrow, 118; Snow Bunting, 1. (Add: Horned Lark, Dec. 23, 25, 26.)-Boswell Belcher (compiler), Margaret Belcher, Mr. and and Mrs. S. R. Belcher.

ERINFERRY, Dec. $26 ; 4$ species, 16 individuals. Hairy Woodpecker, 2; Blue Jay, 6; Black-capped Chickadee, 5; Evening Grosbeak, 3. (Add: Great Horned Owl, 2, Jan. 1; Snowy Owl, 1; Tlec. 30; Downy Woodpecker, 1, Jan. 1; Plack-billed Magpie, 3, Dec. 29; Raven, , Dec. 29; Bohemian Waxwing, flocks, Jan. 2.)-Mrs. E. A. Dodd.

FORT QU'APPELLE, Dec. 26; temp. $15^{\circ}$; calm; 10 inches of snow; 12 species, 47 individuals. Ruffed Grouse, 1; Sharp-tailed Grouse, 4; Gray Partridge, 8; Snowy Owl, 1; Hairy Woodpecker, 1; Downy Woodpecker, 1; Blue Tay, 5; Black-billed Magpie, 1; Blackcapped Chickadee, 2; White-breasted Nuthatch, 1; House Sparrow, 21; Common Grackle, 1. (Add: Mallard 1, Jan. 2; Ring-necked Pheasant, 4, Jan. 2; Rock Dove, 3, Dec. 29; Robin, 4, Dec. 29; Bohemian Waxwing, 7, Jan. 1; Northern Shrike, 1, Dec. 22; Snow Bunting, 500, Dec. 29.)-Dr. and Mrs. G. D. Barnett, E. Manley Callin (comiler), Richard Carter, Mr. and Mrs. Errol Cochrane, Mr. and Mrs. Bernie deVries, Mr. and Mrs. Wm. Gray, Staney Harrison, Ron Hooper, Winnifred and Geoffrey Louks, Jack and Jay Lowe, Dick Nevard, John Norman, Horace Reed, Mr. and Mrs. S. P. Regan, Joe Rumancik.

HEPBURN, Dec. 28; 30 miles by car; temp. $-10^{\circ}$ to above zero; 2 species, 25 individuals. Gray Partridge, 15; Black-billed Magpie, 10. (Add: Snow Bunting, 32, Dec. 29 and 31.)-_Philip Siemens.

HUDSON BAY, Dec. 27; 20 miles by ar in $2 \frac{1}{2}$ hours; temp. $-25^{\circ}$; wind $\mathrm{W}$ $\mathrm{mph}$; clear; 6 species, 300 indiiduals. Black-billed Magpie, 5; Com- mon Raven, 4; Northern Shrike, 1; House Sparrow, 5; Slate-colored Junco, 1; Snow Bunting, 284. (Add: Evening Grosbeak, 4, Jan. 3; Pine Grosbeak, 25, Dec. 28.)-Eldon and Gwen Thorson.

INDIAN HEAD, Dec. 27; 30 miles by car in 1 hour and 6 miles on foot in 5 hours plus 3 miles by horse and sleigh in $1 \frac{1}{2}$ hours and around 4 farm yards for 1 hour; temp. $-5^{\circ}$ to $+5^{\circ}$; wind SE 10 to $20 \mathrm{mph}$; clear; 30 inches of snow; 14 species, 346 individuals. Mallard, 40; Ruffed Grouse, 1; Sharptailed Grouse, 8; Gray Partridge, 11; Rock Dove, 32; Great Horned Owl, 1; Hairy Woodpecker, 4; Downy Woodpecker, 6; Blue Jay, 2; Black-billed Magpie, 22; Black-capped Chickadee, 36; Robin, 1; House Sparrow, 175; Snow Bunting, 7.-Beth Copithorn, Ian Gray, Richard Gray, Bobby Jewitt, Anna Macial (members of the Indian Head 4H Junior Sportsman Club), Carol Beaulieu, Janet Gray (club leaders), Mr. and Mrs. Jim Lang, Brian Scott, Glen Scott, Elaine Scott, Lorne Scott (compiler), Reg. Scott, Gary Seib, Mr. and Mrs. Fred Skinner, Gordon Willerth, Jackie Willerth.

KELVINGTON, Jan. 2; 4 miles by car and around the farmyard in $1 \frac{1}{2}-2$ hours; temp. $-2^{\circ}$; dull and cloudy; 2 feet of snow; 8 species, 151 individuals. Ruffed Grouse, 2; Sharp-tailed Grouse, 1; Downy Woodpecker, 1; Black-billed Magpie, 10; Black-capped Chickadee, 5; Bohemian Waxwing, 45; Starling, 2; Snow Bunting, 85.-Dianne Sloan.

KENASTON, Dec. $31 ; 1 \frac{1}{2}$ hours by truck about the farm area, and half mile about the field and the yard; temp. $5^{\circ}$ to $17^{\circ}$; clear; calm; $12^{\prime \prime}$ of snow; 3 species, 57 individuals. Gray Partridge, 27; Black-billed Magpie, 5; House Sparrow, 25. (Add: Sharp-tailed Grouse, 16, Dec. 29; Rock Dove, 8, Dec. 23; Great Horned Owl, 1, Dec. 20; Horned Lark, Dec. 16, Jan. 2; Snow Bunting, 3000, Dec. 27.)-Lawrence Beckie and family. 
LEADER, Dec. 27; 3 hours about the farm; temp. $20^{\circ}$; clear and calm; 14 inches of drifted snow; 7 species, 67 individuals. Sharp-tailed Grouse, 12; Ring-necked Pheasant, 24; Gray Partridge, 8; Black-billed Magpie ,5; Blackcapped Chickadee, 1; Bohemian Waxwing, 15; House Sparrow, 2. (Add: Snow Bunting, 100, Dec. 28.)-Daisy D. Meyers.

MONTREAL LAKE-LARONGE, Jan. 3 ; 100 miles by car in 2 hours; temp. $0^{\circ}$; wind NW $10 \mathrm{mph}$; partly cloudy with occasional snow flurries; 4 species, 100 individuals. Great Horned Owl, 1; Downy Woodpecker, 1; Common Raven, 91; Black-capped Chickadee, 7. (Add: Black-billed Magpie, 4; Snow Bunting, 47.)-Agatha Harder, Margaret Siemens (compiler), Phyllis Siemens.

MOOOSE JAW, Dec. 26; 71 miles by car and 11 miles on foot; temp. $12^{\circ}$ to $20^{\circ}$; clear and calm; 6 inches of snow on level; 11 species, 1110 individuals. Sharp-shinned $\mathrm{Hawk}, 1$; Sharp-tailed Grouse, 8; Ring-necked Pheasant, 3; Gray Partridge, 25; Rock Dove, 289; Blue Jay, 1; Black-billed Magpie, 51; Black-capped Chickadee, 4; Bohemian Waxwing, 31; Starling, 106; House Sparrow, 591. (Add: Pigeon Hawk, 1, Dec. 24; Snowy Owl, 1, Dec. 31.) -John Horton, Pam Kern, Pat Kern, Leith and $\mathrm{Cy}$ Knight, Peter Muirhead, Murdoch Nelson, David Robinson (Leith Knight, compiler).

NAICAM, Dec. $24 ; 8$ miles by snowmobile; temp. $0^{\circ} ; 6$ species, 46 individuals. Ruffed Grouse, 6; Sharp-tailed Grouse, 17; Hairy Woodpecker, 1; Black-billed Magpie, 3; Black-capped Chickadee, 4; House Sparrow, 15 (Add: Gray Partridge, 2, Dec. 21.) Ronald Jensen.

NIPAWIN, Dec. 27 ; 5 hours on foot and 54 miles by car; temp. $-26^{\circ}$; wind $\mathrm{N} 10 \mathrm{mph} ; 17$ species, 262 individuals. Sharp-tailed Grouse, 5; Rock Dove, 7; Pileated Woodpecker, 1; Hairy Woodpecker, 1; Downy Woodpecker, 3; Gray
Jay, 2; Blue Jay, 3; Black-billed Magpie, 13; Common Raven, 6; Blackcapped Chickadee, 3; White-breasted Nuthatch, 2; Robin, 1; Bohemian Waxwing, 100; House Sparrow, 19; Evening Grosbeak, 7; Pine Grosbeak, 10; Snow Bunting, 82. (Add: Pigeon Hawk, 1, Dec. 22; Willow Ptarmigan, 2, Jan. 3; Gray Partridge, 6, Dec. 28.)-Vern Gunnlaugson Walter and Billy Matthews, Stan Riome.

OUTLOOK Dec. 29; 70 miles by car; temp. $-25^{\circ}$; sunny; about $12^{\prime \prime}$ snow; 6 species, 1018 individuals. Gray Partridge, 8; Rock Dove, 4 ; Horned Lark, 1; Black-billed Magpie, 4; House Sparrow, 1; Snow Bunting, 1000.-Harold Kvinge.

PIKE LAKE, Dec. 31; 3 miles on foot and 54 miles by car in 7 party hours; temp. $6^{\circ}$ to $13^{\circ}$; wind SE $3 \mathrm{mph}$; partly cloudy; 13 species, 376 individuals. Sharp-tailed Grouse, 8; Ringnecked Pheasant, 4; Gray Partridge, 26; Rock Dove, 7; Hairy Woodpecker, 3; Downy Woodpecker, 3; Blue Jay, 6; Black-billed Magpie, 126; Black-capped Chickadee, 31; Robin, 28; Bohemian Waxwing, 6; House Sparrow, 120; Snow Bunting, 8. (Add: Short-eared Owl, 1, Jan. 2; Common Crow, 1, Jan. 2.)-Dr. and Mrs. J. B. Gollop, Mrs. L. Hoyte, J. F. Roy, J. A. Wedgwood.

PRINCE ALBERT, Dec. 29; at feeder in back yard 4 miles north of Prince Albert; 9 species, 134 individuals. Hairy Woodpecker, 2; Downy Woodpecker, 2; Blue Jay, 12; Black-billed Magpie, 2; Common Raven, 3; Blackcapped Chickadee, 12 ; Bohemian Waxwing, 80; Evening Grosbeak, 18; Pine Grosbeak, 8 (Add: Sharp-tailed Grouse, Dec. 23; Snow Bunting, Dec. 23.) Mr. and Mrs. A. O. Aschim.

RAYMORE, Dec. 29; 6 miles on foot in 3 hours and 9 miles by car in 1 hour; temp. $20^{\circ}$; wind E 10 to $15 \mathrm{mph}$; clear and sunny; 12 inches snow on ground; 8 species, 87 individuals. Rock Dove, 21; Great Horned Owl, 2; Snowy Owl, 1; Downy Woodpecker, 1; Black-billed 
Magpie, 14; Black-capped Chickadee, 9; Bohemian Waxwing, 12; House Sparrow, 27. (Add: Ruffed Grouse, 10, Jan. 1; Sharp-tailed Grouse, 9, Dec. 31; Gray Partridge, 10, Dec. 23; Hairy Woodpecker, 1, Dec. 24; Robin, 4, Dec. 22; Pine Grosbeak, 1, Dec. 22, Dec. 25; Common Redpoll, 1, Dec. 26; Snow Bunting, 70, Dec.23.)-Craig Cameron, Wayne Harris (compiler).

REGINA, Dec. 26; 375 miles by car in 24 hours, and 32 miles on foot in 20 hours; temp. $0^{\circ}$ to $15^{\circ}$; wind NW 5-15 mph; sky clear; 30" snow cover; 33 species, 3293 individuals. Eared Grebe, 1; Mute Swan, 7; Whistling Swan, 5; Canada Goose, 600; Mallard, 400; Pintail, 1; Redhesd, 4; Canvasback, 4; Lesser Scaup, 6; Common Goldeneye, 11; Ruddy Duck, 5; Gray Partridge, 37; American Coot, 4; Rock Dove, 297; Great Horned Owl, 1; Snowy Owl, 7; Yellow-shafted Flicker, 3; Red-shafted Flicker, 1; Hairy Woodpecker, 1; Downy Woodpecker, 2; Horned Lark, 2; Black-billed Magpie, 59; Blackcapped Chickadee, 10; Robin, 2; Bohemian Waxwing, 19; Cedar Waxwing, 30; Northern Shrike, 3; Starling, 48; House Sparrow, 1400; Red-winged Blackbird, 1; Brewer's Blackbird, 1; White-throated Sparrow, 1; Snow Bunting, 320.-Gary Anweiler, Jessie Bailey, Fred Bard, Margaret Belcher, Al Binnie, Betty Binnie, Greg Bobbitt, Iola Crouse, Betty Cruickshank, Mr. Bryn Davies, Mrs. Bryn Davies, Wayne Davis, Dan de Vlieger, Lucy Eley, Doug Gilroy, Jackie Hamel, Jim Hines, Hugh Jowsey, Jim Jowsey, Shirley Jowsey, Shirley Larmour, George Ledingham, Margot Lowe, Helen Morrison, Sally Moss, Connie Pratt, Bob Rafuse, Maureen Rever, Joe Roberts, Lorne Scott (compiler), Grace Tubello, Elisabeth Wagner, Jeanie Wagner, Billy Wallace, Holly Wallace, W. J. Wallace, Janie Wilhelm.

SASKATOON, Dec. 26; 251 miles by car in 40 hours and 25 miles on foot in $23 \frac{1}{2}$ hours; temp. $3^{\circ}$ to $13^{\circ}$; wind W 11 to $0 \mathrm{mph}$; clear; 9 inches of snow; 28 species, 6891 individuals. Mallard, 25; American Widgeon, 1;
Canvasback, 2; Common Goldeneye, 10; Pigeon Hawk, 1; Ruffed Grouse, 2; Sharp - tailed Grouse, 25; Ringnecked Pheasant, 5; Gray Partridge, 532; Rock Dove 1133; Great Horned Owl, 3; Snowy Owl, 4; Yellow-shafted Flicker, 5; Hairy Woodpecker, 5; Downy Woodpecker, 7; Horned Lark, 1; Blue Jay, 25; Black-billed Magpie, 262; Black-capped Chickadee, 46; Robin, 44; Bohemian Waxwing, 257; Cedar Waxwing, 50; Northern Shrike, 2; Starling, 17; House Sparrow, 4333; Hoary Redpoll, 1; Common Redpoll, 3; Snow Bunting, 90. - Mark Abley, Michael Bantjes, Rod Bantjes, Bob Besant, Jeff Besant, Joan Besant, Bernard Bisha, Ed Bisha, Raymond Bisha, Hans Blokpoel, Herman Boerma, Philip Boerma, Ricky Buchner, Pern Cordery, Ewen Coxworth, Morley Coxworth Geoff Galloway, Muriel Galloway, Marie Gillespie, J. B. Gollop, M. F. Gollop, Arnie Guenther, Scott Hale, Bert Hardy, Dr. C. J. Houston, David Houston, Donald Houston, Mary Houston, Stuart Houston, Grev. Jones, Caroline Kindrachuk, Jeff Krolik, Mrs. J. McRobbie, Wm. Nickel, David Nowosad, J. Frank Roy, John Shadick, Stan Shadick, James A. Slimmon, Alan R. Smith, Laura Smith, Tom Smith, Jas. A. Wedgwood, Jarrell Wenger.

SKULL CREEK, Dec. 26; 13 species, 176 individuals. Golden Eagle, 1; Sharp-tailed Grouse, 21; Ring-necked Pheasant, 4; Gray Partridge, 5; Rock Dove, 8; Great Horned Owl, 1; Hairy Woodpecker, 4; Downy Woodpecker, 3; Black-billed Magpie, 40; Black-capped Chickadee, 6; House Sparrow, 21; Common Redpoll, 50; Snow Bunting, 12.-Cindy and Jim Benneto, Henry Borman, Austin Drever, Johnny Drever, Dorothy Ecclestone, Phyllis Flaig, Robert, Betty and Patti Mann, Mrs. S. A. Mann (compiler), Helen and Ray Schuler, Peter Swain.

SPIRIT LAKE, Jan. $3 ; 4$ miles in $2 \frac{1}{2}$ hours on foot and 53 miles in $3 \frac{1}{2}$ hours by car; temp. $-12^{\circ}$ to $5^{\circ}$; wind WNW $15 \mathrm{mph}$; clear; 14 inches of snow; 11 species, 324 individuals. Ruffed Grouse, 2; Rock Dove, 4; Great Horned Owl 1; 
Snowy Owl, 1; Pileated Woodpecker, 1; Hairy Woodpecker, 3; Downy Woodpecker, 6; Black-billed Magpie, 8; Black-capped Chickadee, 13; House Sparrow, 105; Snow Bunting, 180. (Add: Robin, 1, Dec. 23 through 27; Bohemian Waxwing, 1, Jan. 2.)-Wm. Anaka, Mrs. Gunn, Joyce Gunn, Frank Switzer.

SQUAW RAPIDS-NIPAWIN, Dec. 26; 96 miles by car; temp. $0^{\circ}$; wind $\mathrm{N} 10$ $\mathrm{mph} ; 9$ species, 610 individuals. Mallard, 2; Common Goldeneye, 11; Bald Eagle, 2; Snowy Owl, 1; Hawk Owl, 1; Black-billed Magpie, 10; Common Raven, 23; Bohemian Waxwing, 30; Snow Bunting, 530.--Vern Gunnlaugson, Paul Riome, Stan Riome.

TUBEROSE, Dec. 26 ; temp. $25^{\circ}$ to $30^{\circ}$; clear, sunny; no wind; 2 species. Gray Partridge, flock; Snowy Owl,1.Cliff Matthews.

VALLEY CENTRE, Dec. 27; 77 miles by car in $3 \frac{1}{2}$ hours and 2 miles on foot in $1 \frac{1}{2}$ hours; temp. $12^{\circ}$; clear, sunny calm; 8" snow; 8 species, 1017 individuals. Sharp-tailed Grouse, 13; Ringnecked Pheasant, 2; Gray Partridge, 39; Rock Dove, 71; Great Horned Owl, 1; Black-billed Magpie, 75; House Sparrow, 364; Snow Bunting, 452. (Add: Peregrine Falcon, 1, Dec. 31; Snowy Owl, 1, Dec. 26 and 31.)Blaine Lacina, Don, Ron and Wayne Renaud.

VAL MARIE, Dec. 24; 35 miles by vehicle and 2 on foot along the Frenchman River; 5 species, 128 individuals. Sharp-tailed Grouse, 1; Ring-necked Pheasant, 1; Horned Lark, 25; Blackbilled Magpie, 1; House Sparorw, 100. (Add: Golden Eagle, 1, Dec. 26; Sage Grouse, 2, Dec. 27; Gray Partridge, 5, Dec. 27; Snowy Owl, 1, Dec. 27; Shorteared Owl, 2, Jan. 2.) - J. David Chandler.

WAUCHOPE, Dec. $26 ; 4$ miles by car and $3 \frac{1}{2}$ miles on foot in $4 \frac{1}{2}$ hours plus short observations at the feeding station; temp. $29^{\circ} \max$ and $18^{\circ} \mathrm{min}$; clear and sunny; wind light; 7 inches of snow; 8 species, 378 individuals. Rock Dove, 16; Hairy Woodpecker, 3; Downy Woodpecker, 1; Black-billed Magpie, 1; Black-capped Chickadee, 6; Robin, 1; House Sparrow, 100; Snow Bunting, 250. (Add: Ruffed Grouse, 1, Dec. 31; Sharp-tailed Grouse, 15, Jan. 2.)-Dale Hjertaas.

WELLI NGTON LAKE, $(25$ miles northwest of Eldorado, Sask.) Dec. $22 ; 6$ species, 25 individuals. Willow Ptarmigan, 15; Hairy Woodpecker, 2; Black-backed Three-toed Woodpecker, 1; Gray Jay 6; Common Raven, 3; Black-capped Chickadee, 2. (Add: Common Redpoll, 25, Dec. 30.)-Mrs. E. A. Middleton.

YORKTON, Dec. $26 ; 8$ miles in 8 hours on foot, and 107 miles in 4 hours by car; temp. $13^{\circ}$; wind NW $20 \mathrm{mph}$; clear with ground drift; 18" snow heavily drifted on fields; 8 species, 513 individuals. Pigreon Hawk, 1; Sharp-tailed Grouse, 11; Rock Dove, 25; Black-billed Magpie, 12; Blackcapped Chickadee, 1; House Sparrow, 175; Pine Grosbeak, 1; Snow Bunting, 287. (Add: Gray Partridge, 2, Dec. 27.)-Grant Black, Harry and Keith Monette, Frank Switzer (compiler).

BANGOR (This report was received late and without a date). 8 species, 140 individuals. Ruffed Grouse, 2; Hairy Woodpecker, 1; Downy Woodpecker, 1; Gray Jay, 1; Black-billed Magpie, 3; Black-capped Chickadee, 4; House Sparrow, 3; Snow Bunting, 125 Mrs. A. Thompson.

FORT SMITH, N.W.T., Dec. 28; 65 miles by car in $3 \frac{3}{4}$ hours plus around the town of Fort Smith; temp. $-10^{\circ}$; wind E. $5 \mathrm{mph}$; mostly sunny with some light overcast; 8" snow; 5 species, 115 individuals. Rock Dove, 12; Gray Jay, 2; Common Raven, 98; Boreal Chickadee, 1; White-winged Crossbill, 2. (Add: Gyrfalcon, 1, immature, Dec. 25.) - Ernie, Elsie and Pamela Kuyt. 